\title{
Conflictos, controversias y problemas públicos. El caso de los ferrocarriles metropolitanos de Buenos Aires en la prensa (2002-2015)
}

\author{
Conflicts, disputes and public problems. The case of the Buenos \\ Aires Metropolitan Railways in the print media (2002-2015)
}

\author{
https://doi.org/10.22235/d33.2373
}

\author{
Candela Hernandez
}

ORCID: 0000-0002-3528-9479

\begin{abstract}
Instituto de Investigaciones Gino Germani, Universidad de Buenos Aires.
\end{abstract}
Consejo Nacional de Investigaciones Científicas y Técnicas, Argentina.

\begin{abstract}
RESUMEN
El objetivo del presente artículo es dar cuenta del proceso de construcción del sistema ferroviario de pasajeros de la Región Metropolitana de Buenos Aires (RMBA) como problema público en el período 2002-2015. Dicho análisis se aborda al estudiar la evolución que tuvieron los conflictos desarrollados en el interior del sistema y las controversias en torno a su problematización social en la arena pública, a partir del tratamiento dado a la temática en la prensa. Se implementa una estrategia metodológica cuantitativa y cualitativa que combina el uso de fuentes primarias y secundarias. Se sostiene que las perturbaciones del sistema (conflictos y accidentes) coadyuvaron progresivamente a madurar los debates en torno a la definición del problema ferroviario, hasta consolidarlo asociado a la cuestión de la corrupción.

Palabras clave: conflicto social; problema público; arena pública; sistema ferroviario de pasajeros; prensa.
\end{abstract}

\begin{abstract}
The aim of this article is to account for the stages in which the Buenos Aires Metropolitan Area's Railway System for Passengers (RMBA for its Spanish acronym) turns into a public matter for the period 2002-2015. This analysis is approached from the evolution of the conflicts developed within the system and the controversies surrounding its social problematization in the public arena from the treatment given to the subject in the press. A quantitative and qualitative methodological strategy is implemented by combining the use of primary and secondary sources. It is argued that the disturbances of the system (conflicts and accidents) contributed to progressively develop the debate around the definition of the railway issue, strengthening its shaping as a public matter associated with corruption's issues.

Keywords: social conflict; public problem; public arena; railway system of passengers; press.
\end{abstract}




\section{Introducción}

Los trenes urbanos de pasajeros de la Región Metropolitana de Buenos Aires (RMBA) ${ }^{1}$ tienen una función nodal en la estructuración de la movilidad cotidiana de sus usuarios. Este modo de transporte es una vía de comunicación que constituye -para una porción significativa de la población- la principal alternativa disponible para garantizar sus desplazamientos diarios. Durante una gran cantidad de los años comprendidos en el período de estudio, este servicio se caracterizó por brindar una calidad deficiente, observable en condiciones de infraestructura precarias, material rodante degradado, escasa seguridad operativa, viajes hacinados y mínimas garantías de prestación efectiva por demoras, poca frecuencia y cancelaciones de servicio. Estos fueron algunos de los atributos típicos de los ferrocarriles metropolitanos que los convirtieron en territorio de una intensa conflictividad social.

En distintos momentos cobraron protagonismo formas contenciosas de disímil índole y magnitud que -directa o indirectamente- abonaron a que la calidad del servicio cobrara relevancia social. Los pasajeros apedreando estaciones, incendiando formaciones y violentando trabajadores frente a cortes prolongados de servicio; la muerte de un joven militante de un partido político de Izquierda, fallecido en contexto de un conflicto entre trabajadores ferroviarios; el peor accidente de la historia ferroviaria metropolitana y los familiares de las víctimas ${ }^{2}$ movilizados en demanda por justicia fueron los acontecimientos que marcaron la creciente controversia pública generada en torno a la cuestión ferroviaria.

Con el correr del tiempo los debates fueron anudándose en un trayecto que terminó con la consolidación del tema como problema público. Las malas condiciones de viaje, sus implicancias para el transporte de pasajeros, las causas atribuidas y las responsabilidades asignadas a esta situación se convirtieron en uno de los focos de la discusión de la actualidad argentina. Como resultante de este proceso, la corrupción emergió como la variable explicativa que concitó la mayor legitimidad social.
Este artículo se propone dar cuenta de las etapas de la construcción de los ferrocarriles metropolitanos como problema público para el período 2002-2015. La exposición se articula del siguiente modo: como punto de partida se presenta la estrategia metodológica utilizada. En el apartado siguiente se definen los principales lineamientos teóricos para el abordaje del nudo problemático. Posteriormente se profundiza sobre el caso de estudio, caracterizándolo según la evolución de los conflictos desarrollados al interior del sistema por sus implicancias en la instalación pública de la temática. Luego se abordan los debates tal como fueron registrados por la prensa, fuente tomada como insumo para la captura de los discursos circulantes. Finalmente, se presentan las conclusiones.

\section{Estrategia metodológica}

Para avanzar sobre los interrogantes propuestos se recurrió principalmente a distintas bases de datos de elaboración propia, confeccionadas en el marco del proyecto UBACyT "Transporte público y conflicto social”, con sede en el Instituto de Investigaciones Gino Germani de la Facultad de Ciencias Sociales de la Universidad de Buenos Aires (IIGG-UBA).

Los insumos consisten en una base de registros de prensa sistemáticamente recopilados (RDP-IIGG, 2015) y

$1:$ : La RMBA comprende a la Ciudad Autónoma de Buenos Aires (CABA) y a 43 partidos de la Provincia de Buenos Aires (Kralich, 2016). La mayor parte del tendido ferroviario está en este conurbano metropolitano y consta de siete líneas que lo integran: Sarmiento, Roca, Belgrano Sur, Mitre, San Martín, Belgrano Norte y Urquiza. Cuenta con un desarrollo de $894 \mathrm{~km}$ de vías que unen 262 estaciones con 4 grandes terminales ubicadas en la CABA (Constitución, Retiro, Once y Estación Buenos Aires) (Comisión Nacional de Regulación del Transporte, 2017).

2:: El 22 de febrero de 2012 a las 8.33 a.m. una formación del ferrocarril Sarmiento ingresó a la estación terminal de Once y chocó con el sistema hidráulico de amortiguación del andén. El impacto provocó el colapso de la formación y un saldo de 789 heridos y 52 víctimas fatales. Con motivo del accidente se conformó el movimiento de familiares de las víctimas de Once (Hernandez, 2020). 
un catálogo de eventos conflictivos contenciosos (ACC-IIGG, 2015). El relevamiento abarcó desde el 16 de octubre de 2002 -momento en que se dictó el Decreto de Emergencia Ferroviaria N. ${ }^{\circ}$ 2075/02- hasta el 20 de mayo de 2015, cuando fue promulgada la Ley de Ferrocarriles Argentinos N. ${ }^{\circ} 27132$.

La base de datos de prensa incluye la totalidad de las noticias que contenían alguna referencia al sistema de transporte ferroviario metropolitano de pasajeros. Esta fue realizada a partir del diario argentino La Nación (www.lanación.com.ar) que tiene un perfil conservador en términos políticos culturales, ubicado en abierta tensión con la política oficialista del entonces Gobierno Nacional ${ }^{3}$ (Vommaro y Baldoni, 2012; Viotti, 2015). Su elección estuvo fundamentada sobre un estudio preliminar de la cantidad y calidad de las noticias que proveían cada uno de los diarios nacionales (La Nación, Página/12 y Clarín). La base concluyó con un total de 2038 casos.

La elaboración de esta base se funda en la relevancia de los medios de comunicación en la construcción de problemas públicos. Estos tienen una lógica de funcionamiento que propicia la interacción entre distintos ámbitos, reciben el input de expertos y permiten aproximarse al tratamiento dado a un tema en distintos espacios sociales de discusión (Frigerio, 1997). Su tipo de producción coyuntural y diaria se ejercita en la administración de sucesos relativamente previsibles o inesperados que redundan rápidamente en productos circulables (Tuchman, 1973). El resultado de la actividad periodística es la noticia, en la que se producen y reproducen criterios de selección e intelección de la realidad que aportan a la construcción de representaciones de amplia y rápida circulación social (Alsina, 1989; Lorenc Valcarce, 2005b). Este proceso no se produce de un modo azaroso, sino que está atravesado tanto por el posicionamiento de la línea editorial como por los intereses de los grupos económicos y burocracias dominantes con injerencia en el sector (Río Ruiz, 2008). No obstante esta advertencia, Schuster et al. (2006) señalan que las exigencias de legitimidad y credibilidad imponen constricciones a los márgenes para la manipulación de la información por parte de los medios. Esto hace que las notas constituyan un insumo productivo para el trabajo de investigación.

El procesamiento de los datos constó de dos etapas. En la primera, los registros fueron ordenados cronológicamente y codificados según tema. Esto permitió reconocer los acontecimientos destacados en función de la distribución y concentración de las notas en torno a determinados tópicos. De este modo fueron identificados los principales hitos mediáticos registrados por la prensa relativos al sistema ferroviario metropolitano. Otro indicador que robustece la ponderación de los casos es su arribo a las portadas de los diarios. Esta posición colabora a instalar un tema como merecedor de la atención social y de los poderes públicos (Lorenc Valcarce, 2005a). Los conflictos y accidentes ocurridos en los trenes urbanos fueron los que contaron con la cobertura mayoritaria de la prensa analizada. También fueron tapa, tanto de los diarios estudiados como de otros de tirada nacional (Hernandez, 2017).

El objetivo de la segunda instancia de procesamiento fue delinear la estructura narrativa que el problema ferroviario asumió en la arena mediática para reconstruir su evolución. Con base a las herramientas conceptuales provistas por las teorías de los problemas públicos se trabajó sobre los hitos mediáticos identificados en torno a cinco dimensiones: a) emisor b) definición del problema c) causas percibidas d) responsabilidades atribuidas e) posibles soluciones. Con base a éstas se contabilizó según emisor la primera mención hecha sobre cada uno de los puntos b) c) d) y e) en todas las notas, omitiendo las reiteraciones relativas a un mismo tipo que pudieran aparecen en el desarrollo del texto noticioso. Esto permi-

3:: Las presidencias consecutivas de Néstor Kirchner y Cristina Fernández de Kirchner, ambos pertenecientes a la coalición política Frente para la Victoria, abarcaron un período de 12 años al frente del Gobierno del Estado ( 25 de mayo de 2003 - 9 de diciembre de 2015). Por el período en estudio, salvo indicación contraria, cada vez que se mencione al Gobierno Nacional se estará haciendo referencia a alguno de sus mandatos. 
tió ponderar los actores intervinientes en las controversias y las formas de estructurar el problema.

También se avanzó en un análisis cualitativo de los argumentos emergidos en la arena mediática en función de las dimensiones arriba señaladas que permitieran reconstruir las narrativas emergentes en torno al caso. Complementariamente se recurrió al diario Página/12, históricamente un medio argentino de centro-izquierda que -a diferencia del anterior- fue afín a los gobiernos kirchneristas (Vommaro y Baldoni, 2012; Viotti, 2015). Los registros provenientes de esta última fuente fueron circunscriptos a los temas previamente reconocidos como relevantes, con el propósito de nutrir y encontrar matices en la construcción problematizante que la perspectiva de este medio podría aportar al análisis.

Por su parte, el catálogo de eventos contenciosos fue elaborado a partir de la base de prensa confeccionada. El recurso de los periódicos como fuente de registro y análisis de distintas formas de disconformidad social tiene una amplia tradición en el campo de los estudios sociológicos dedicados a la protesta y a la acción colectiva (Río Ruiz, 2008). Pueden mencionarse antecedentes locales e internacionales que aplican este método al estudio de la conflictividad social (Marín, 2007; Tilly, 2008; Silver, 2005; Schuster et al., 2006; Programa de Investigación sobre el Movimiento de la Sociedad Argentina, s. f.; Stiberman, 2017). La elaboración de este tipo de catálogos permite reconstruir una serie de largo plazo al reunir episodios dispersos en el espacio y en el tiempo, permitiendo realizar cronologías y caracterizaciones generales del conflicto. Snyder y Kelly (1977) indican un elemento adicional por considerar. Sostienen que la probabilidad de registro de los eventos depende tanto de la lógica de producción que tienen los medios de comunicación como de los atributos de los conflictos. Los episodios de magnitud y de tipo disruptivo ganan en probabilidades de ser reportados. En el caso en estudio, el carácter de los episodios registrados dota una mayor confiabilidad a la serie construida.

En lo que respecta al procesamiento de la información fue necesario generar un corrimiento de la unidad de análisis, que dejó de ser la nota para pasar a situarse en las acciones colectivas contenciosas ${ }^{4}$ ocurridas en el marco de los conflictos en el espacio ferroviario. El tratamiento dado a los datos procuró captar en cada caso la existencia de un actor, forma de acción, meta, destinatario, sincronización temporal (un tiempo de realización) y convergencia en el espacio (un lugar o conjunto de lugares relativamente definidos de la acción). Con base en esta información se realizó una búsqueda de las asociaciones más recurrentes, construyendo de ese modo una tipología de la conflictividad en el transporte ferroviario de pasajeros. La base cerró con 153 eventos, en los cuales los disconformes protagonizaron 194 acciones colectivas contenciosas.

El procesamiento y análisis sistemático de ambos conjuntos de datos permitió establecer correspondencias entre la evolución de los conflictos típicos reconocidos en el marco del servicio ferroviario y los debates públicos más significativos del período para este caso. Como se muestra en el desarrollo del artículo, la resultante de este entrecruzamiento constituye un observable de cuáles fueron aquellos que promovieron procesos de controversia que abonaron a la construcción pública del problema ferroviario.

\section{Precisiones conceptuales}

La construcción de un problema social de carácter público es un proceso colectivo. En este proceso, ciertas condiciones son percibidas y conceptualizadas por un conjunto de actores como nocivas para una porción considerable de la población. Su arista problemática reside en la posibilidad de ser modificadas y sobre las cuales se espera

\footnotetext{
4:: Las acciones colectivas contenciosas pueden adquirir diversas formas o performances con las que los disconformes expresan sus demandas o reivindicaciones (Tilly, 2000). Componen el denominado repertorio de acción y nutren el acervo de prácticas de las que disponen los actores en conflicto. Los repertorios están históricamente constituidos derivados de la experiencia de lucha y pueden variar entre tiempos históricos y sociedades o en su interior (Tilly, 2000, 2008). Son ejemplos perfomances típicas como la huelga, movilización, actos, entre otras.
} 
que se ejecuten acciones que intervengan en la dirección de solucionarlas (Loseke, 2007). En distintos momentos y contextos existe una heterogeneidad de situaciones concebidas como negativas, extendidas, transformables y sobre las cuales debería intervenirse. No obstante, pese a que determinadas circunstancias puedan entrar en las categorizaciones previas, estas no necesariamente se transforman en epicentro de conflicto o controversia en el ámbito público (Gusfield, 1981/2014). Para que esto suceda deben convertirse en asunto de reflexión y objeto de la protesta al tiempo que en recurso y propósito para la acción pública (López, 2008, p. 5).

En este movimiento existen distintas arenas -medios de comunicación e industrias culturales, órganos de representación política, comunidad cientifica, entre otras (Hilgartner y Bosk, 1988; Frigerio, 1997)- en las que se publicitan determinadas problemáticas sociales. Al convertirse estas en objetos de atención y discusión, las representaciones circulantes en torno a ellas resultan variadas y son heterogéneas las narrativas que buscan estructurar el problema en disputa. En ocasiones también pueden ser incluso incoherentes o contradictorias entre sí. Ahora bien, en la medida en que un tema transita por distintos ámbitos y estos logran articularse en función de caracterizarlo, contribuyen a amplificar la atención pública sobre determinadas cuestiones e intervienen en su consolidación problemática. Como se destacó en el apartado anterior, los medios de comunicación tienen un rol importante en este proceso. Desde su propia especificidad, están legitimados por el mercado y por los sectores simbólicamente privilegiados para resaltar los temas que van a ser considerados relevantes en un contexto específico. Se conforman como escenario destacado para el desarrollo de las controversias que giran en torno a ciertos temas, con una capacidad de llegada a una variedad y multiplicidad de públicos (Frigerio 1997; Lorenc Valcarce, 2005b; Hernandez y Saferstein, 2018).

De este proceso participa una diversidad de actores socialmente significativos, que intervienen y se movilizan en torno a la problematización de un determinado tema de acuerdo con sus recursos materiales y simbólicos acumulados. Estos últimos redundan en una mayor o menor capacidad de imponer visiones del mundo, lo cual expresa jerarquías de credibilidad y legitimidad entre quienes intervienen en la definición de un problema como tal (Langbehn, 2015; Schillagi, 2011, Loseke, 2007).

En este sentido aparece la cuestión de la propiedad y desentendimiento de la propiedad que está, en gran medida, relacionada al poder y la autoridad que pueden mostrar los grupos y las instituciones para ingresar o mantenerse afuera de las discusiones en la arena pública. En este abanico están presentes dos responsabilidades: causal y política (Gusfield, 1981/2014, p. 79). La primera refiere a una explicación fáctica de la secuencia de situaciones que desencadenan un problema. La segunda apunta a la identificación de quienes son los encargados de hacer algo respecto. Gusfield (1981/2014) denomina a esta como una “cuestión de políticas". Como refuerza Langbehn (2015), siguiendo a Gusfield, el hecho de interpretar una situación como problema público lleva implícita una demanda al Estado, en la medida que se supone que el problema es transformable o erradicable por la acción estatal (p. 31).

Al mismo tiempo, como señala Lorenc Valcarce (2005a), independientemente de los fines oficiales que tenga la implementación de determinadas políticas, estas además concitan una dimensión simbólica. En la medida en que intervienen sobre determinado nudo problemático, no sólo lo reconocen como tal, sino que el tipo de acciones para remediarlo contribuyen al proceso de definición del problema. El desenlace que un determinado tema encuentra en su trayectoria problematizante sucede cuando las múltiples narrativas que circulan en torno a él convergen vinculadas a determinadas categorías. Estas contribuyen a delimitar su conceptualización como problema público, que momentáneamente se estabiliza en una acepción dominante que ordena su estructura (Pereyra, 2013). Para el caso en estudio, la perspectiva utilizada permite reconstruirla a partir de cinco preguntas que no por su sencillez carecen de complejidad: ¿Cuál es el problema? ¿Cuáles son sus causas? ¿Quiénes son los responsables? 
Gráfico 1: Cantidad de acciones colectivas conflictivas según actor. Sistema ferroviario de pasajeros de la RMBA. 2002-2015.



Fuente: Elaboración propia en base al catálogo de acciones colectivas conflictivas IIGG. N=73.

¿Qué soluciones pueden promoverse? ¿Quiénes tienen la capacidad de construir consenso público sobre las atribuciones asignadas en sus respuestas a estos interrogantes?

\section{Trenes urbanos: escenario de conflicto}

Para desentrañar el planteo propuesto, primero es necesario avanzar en la caracterización de los conflictos ocurridos en los trenes urbanos. Diversos fueron los episodios protagonizados por una multiplicidad de actores que se expresaron bajo una heterogeneidad de formas de acción colectiva contenciosa. Estas tuvieron distintos niveles de institucionalización, capacidad disruptiva, innovación y violencia (Rebón y Hernandez, 2016).

Con independencia de la variedad que la conflictividad asumió en el período, pudieron reconocerse modos típicos de su desarrollo. Como muestra el Gráfico 1, son tres los que se destacan por su relevancia en distintos momentos: ataque de pasajeros, cortes de vías o bloqueos de boleterías de trabajadores tercerizados y actos de familiares de las víctimas 5 . También, como se verá más adelante en el Gráfico 2, esta evolución se corresponde con la circulación de los discursos públicos en torno a los episodios registrados por la prensa que intervienen en la estructuración del problema público ferroviario.

Los pasajeros de los trenes metropolitanos emergieron durante los primeros años analizados como una de las principales figuras del conflicto. El Gráfico 1 muestra que entre octubre de 2002 y mayo de 2015 ocurrieron un total de 26 ataques. En su distribución, estos tendieron a concentrarse en el período 2005-2008 con el 62\% de los casos (ACC-IIGG, 2015). Las acciones de disconformidad protagonizadas por pasajeros presentaron como rasgo distintivo el avance colectivo violento contra objetos y trabajadores de las empresas prestatarias, existiendo una estrecha relación entre el blanco del ataque y los motivos desencadenantes del hecho. Su dinámica tuvo un carácter espontáneo, en el que no se identificaron demandas formuladas de manera articulada, pero que fueron atribuidas a la disconformidad con el servicio (Pérez, 2013). Los episodios más destacados fueron los ocurridos en la Línea Sarmiento en la estación Haedo (2005); la Línea Roca en la estación Constitución (2007) y nuevamente en la Línea Sarmiento en la estación

5:: La acción colectiva contenciosa protagonizada por los sindicatos ferroviarios no se incluye en la serie graficada porque, aunque compone una parte significativa de la serie de conflictividad (31\%), presenta una distribución homogénea en el tiempo que no alcanza picos (ver Rebón y Hernandez, 2016). Los sindicatos no aparecen como protagonistas destacados de algunas de las etapas trabajadas en la construcción de los trenes urbanos como problema público. Incluso en la prensa la confrontación capital-trabajo tampoco alcanza los niveles de repercusión y cobertura de los otros casos (excepto el de los trabajadores tercerizados). Puede hipotetizarse que el alto grado de institucionalización de la acción sindical, vía el derecho a huelga, torne a estas acciones en episodios de menor noticiabilidad, desde la lógica de producción de los medios. 
Castelar (2008). Estos casos particulares sobresalieron por los niveles de violencia, la cantidad de participantes, la duración de los hechos y el enfrentamiento con las fuerzas de seguridad (Pérez, 2013).

Trascendido el protagonismo de los pasajeros, los trabajadores tercerizados tomaron relevancia. Al ocupar una posición periférica a la producción del servicio, por el tipo de tareas que realizan, estos necesitaron de una fuerte impronta disruptiva en sus acciones, que tomaron principalmente la forma de corte de vías, bloqueo de boleterías o liberación de molinetes y corte de calles. Como se aprecia en el Gráfico 1, produjeron un total de 23 acciones, concentradas entre 2010 y 2011, lo que representa el 61\% de las registradas en el período para este actor (ACC-IIGG, 2015). Entre sus demandas se distinguieron aquellas vinculadas al pase a planta permanente y contra los despidos de trabajadores. Este eje de confrontación estuvo trazado en oposición a las empresas prestatarias, al Poder Ejecutivo Nacional y a los sindicatos del sector, configurados como un actor adverso a sus intereses (ACC-IIGG, 2015).

En el período estudiado los principales gremios ( $\mathrm{La}$ Fraternidad y la Unión Ferroviaria) encontraron un escenario favorable en el mercado para la negociación de las condiciones de los asalariados (Etchemendy y Collier, 2008; Antón, Castro, Rebón y Salgado, 2011). En particular, en el servicio de los trenes urbanos tuvieron una posición estratégica con el arribo de sus referentes a puestos ejecutivos en la cartera de transporte y en las empresas ferroviarias estatales (Pérez y Rebón, 2017). Esta situación los dotó de una amplia disponibilidad de recursos, observable en una mejora de las condiciones de explotación de la fuerza de trabajo sindicalizada a expensas de excluir de los beneficios a otros colectivos de trabajadores como los tercerizados ferroviarios (Centro de Estudios Legales y Sociales, 2012; Basualdo, Morales y Cabello, 2014). El caso más significativo de esta etapa sucedió el 20 de octubre de 2010. Ese día un grupo de choque de corte sindical atacó con armas de fuego a trabajadores subcontratados de la Línea Roca que estaban manifestándose con un corte de vías junto a otros colectivos sociales que apoyaban su reclamo. El saldo del evento fue de un muerto (Mariano Ferreyra, un joven militante del Partido Obrero) ${ }^{6}$ y tres heridos de gravedad. ${ }^{7}$

En el año 2012 cambió nuevamente el eje de la contienda. Los familiares de las víctimas de Once ganaron protagonismo como figura del conflicto. Estos se posicionaron como un activo actor demandante de justicia y de mejoras en la calidad del servicio. En lo que respecta a las acciones colectivas, la modalidad característica de este agrupamiento fueron los actos. En total se registraron 24 de este tipo. Los ejes de su confrontación se ordenaron con el Gobierno Nacional -a quien le atribuyeron responsabilidad en el acontecimiento- y el Poder Judicial como actor interviniente en el esclarecimiento del caso (ACC-IIGG, 2015).

La evolución de la conflictividad reseñada muestra que, en los momentos de mayor intensificación, ocurrieron episodios que por su magnitud destacaron por sobre el resto. Esta diferenciación no sólo estuvo dada por los efectos en la alteración del orden en el ámbito de la producción y la realización del servicio, con consecuencias directas sobre la infraestructura, el material rodante y su normal funcionamiento. Estos lograron traspasar la perturbación que produjeron al interior del sistema de los trenes metropolitanos, tomando el carácter de acontecimientos (Schillagi, 2011). La emergencia pública de los hechos abrió la disputa a un conjunto de actores sociales y políticos que pasaron a formar parte de la elaboración colectiva de las representaciones circundantes y de los temas que instalaron, contribuyendo a la apertura de un proceso de controversia hasta arribar a su maduración como problema público.

6:: El Partido Obrero (P0) es una fuerza política argentina de izquierda con una fuerte cultura de cooperación en distintas formas de expresión colectiva.

7:: El caso Ferreyra operó como un elemento dinamizador del conflicto de los tercerizados. Sucedido el hecho, se empezaron a incorporar trabajadores a la planta de la empresa contribuyendo, al menos momentáneamente, a la distensión de conflicto. 
Gráfico 2: Promedio mensual de noticias por año y acontecimientos destacados según intensidad en el conflicto o magnitud del hecho. Sistema ferroviario de la RMBA. 2002-2015.



Fuente: Elaboración propia con base en registro prensa IIGG (2015). N=2038

Trenes urbanos como problema

Con el transcurso de los años los ferrocarriles metropolitanos tomaron relevancia en la atención recibida por la prensa y por los medios de comunicación en general. En este marco la premisa propuesta por Snyder y Kelly (1977) -que atiende a la noticiabilidad de los episodios disruptivos- resulta observable en que los conflictos destacados por este carácter fueron los que aparecieron con mayor fuerza en la escena pública. Estos alcanzaron la portada de los diarios de mayor tirada a nivel nacional. Entre los varios titulares por entonces publicados, pueden citarse como ejemplo: "Furia y Caos por atraso de trenes" (Clarín, 16 de mayo de 2007) o "Un tren explosivo" (Página/12, 05 de septiembre de 2008) para atender a los ataques de pasajeros. "A sangre fría” (Vales, Página/12, 21 de octubre de 2010) o "Fuerte conmoción por el asesinato de un militante" (Clarín, 21 de octubre de 2010) para referir al caso de Mariano Ferreyra. "Estación Horror" (Página/12, 23 de febrero de 2012) o "Tragedia anunciada" (Clarín, 23 de febrero de 2012) para el acontecimiento de Once.

También como muestra el Gráfico 2, estos episodios generaron picos en la cobertura mediática. Su medición, con base en el promedio mensual de noticias, permite identificar una variación conjunta entre ambas dimensiones - conflictos, accidentes y notas de prensa(Hernandez, 2017).
La cobertura de la prensa sobre la cuestión ferroviaria en estos años estuvo mayoritariamente concentrada en torno a la calidad del servicio (62\%). ${ }^{8}$ Entre las noticias nucleadas por referir directamente a este tema, los reclamos de pasajeros bajo la modalidad de ataques fueron el principal foco de la cobertura mediática. Como puede apreciarse en el Gráfico 2, la frecuencia de notas relativas al caso de interés tendió a intensificarse, aumentando su promedio mensual con picos que encontraron correspondencia con los ataques señalados como más relevantes. Para 2007, el promedio de notas fue de 9,3 puntos, el más alto para el subperíodo referido. Además, alcanzó al 60\% de las notas de la agrupadas en torno a la calidad del servicio, que continuó como temática dominante (RDP-IGG, 2015). En el año 2010, la calidad como tema principal fue desplazada al segundo puesto por el caso de Mariano Ferreyra. El 50\% de las notas relativas al servicio ferroviario registradas en la base de datos para ese año abordaron este caso (RDP-IIGG, 2015). El promedio mensual de noticias alcanzó para 2010 la media de 11,1 y de 16 puntos en el año siguiente.

8:: La calidad de servicio agrupa a las noticias referidas a las condiciones de prestación, accidentes, los reclamos vinculados a ambos y los subsidios al sistema. Las categorias restantes incluyen a las condiciones laborales (19\%); obras de infraestructura (5\%); cambios en la organización social del servicio (4\%); relación del gobierno con las empresas prestatarias (3\%); anuncios (3\%); regulaciones $(1 \%)$; vandalismo (1\%); tarifas (1\%); otros (1\%). 
En 2012 el promedio de noticias publicadas sobre el sistema ferroviario duplicó a las del año anterior, pasando de 16 a 37 noticias mensuales promedio. El acontecimiento de Once tuvo un peso sustantivo en la distribución temática de las notas registradas, que alcanzó al 67\% de los casos. Entre estas la mayoría estuvo vinculada al caso de Once y al problema de la corrupción en el servicio (RDP-IIGG, 2015). En el año 2013 fue relevada una media de 43 noticias mensuales. Dentro de este agrupamiento los distintos accidentes tuvieron una proporción mayoritaria (64\%), no sólo en relación con el caso de Once, sino también a los ocurridos en ese año en las estaciones de Once y Castelar (RDP-IIGG, 2015). En 2014 el juicio por la tragedia de Once reinstaló con fuerza en la agenda mediática el problema ferroviario que alcanzó al 67\% de las notas registradas en la base de datos para ese año.

Los ataques de pasajeros operaron como acontecimientos que dispararon las primeras controversias. Distintos actores (empresas prestatarias, sindicatos, funcionarios del Gobierno Nacional y referentes de partidos opositores) comenzaron a participar en la emergencia de los ferrocarriles como objeto de debate, articulando sus discusiones en torno a la calidad del servicio. En una primera instancia, entre ellos existió un consenso en reconocer y catalogar como negativas a las condiciones de viaje ofrecidas. En segundo lugar, las causas y responsabilidades atribuidas a tal situación comenzaron a vincularse a la política de subsidios del Gobierno Nacional ${ }^{9}$, al incumplimiento de las obligaciones contraídas por las empresas prestatarias y la laxitud de controles y la escasa efectivización de sanciones por parte de la Comisión Nacional de Regulación del Transporte $(\mathrm{CNRT})^{10}$.

Se identificó una embrionaria orientación a focalizarse en el accionar de las empresas prestatarias, señaladas por los pasajeros como responsables (a través de los ataques) y por los sindicatos y el Gobierno Nacional. Esta lectura fue reforzada por la respuesta estatal que avanzó en las primeras cancelaciones de contrato a una de las cuatro empresas privadas que explotaban los servicios, por incumplir las garantías mínima de presta- ción. El fragmento de prensa presentado a continuación ilustra un observable del carácter de las controversias desplegadas en este momento:

Todo se mezcla cuando sucede algún hecho como el de ayer sobre las vías de los trenes urbanos [a propósito del estallido de Castelar 2008]. Los subsidios para mantener las tarifas bajas, los millones para obras que pocas veces se hacen los concesionarios amigos del poder, el mal servicio y la falta de control, la ausencia de políticas ferroviarias definidas. Todo mezclado (Cabot, 5 de septiembre de 2008, La Nación).

El armado de relaciones entre los distintos actores con participación en la provisión del servicio de trenes contribuyó a configurar las condiciones para la emergencia de otros tipos de conflicto, cuya figura protagonista fueron los trabajadores tercerizados. En este contexto, la muerte del joven Mariano Ferreyra concitó una amplia repercusión mediática, instalando nuevamente al servicio ferroviario de pasajeros en la escena pública. Los debates en torno a este acontecimiento, si bien no se centraron en la calidad del servicio, progresivamente comenzaron a desanudar el urdido de relaciones espurias bajo las que se entendió se encontraba operando el ferrocarril.

9:: Desde el año 2003, en contexto de un esquema tarifario congelado, las erogaciones asignadas por el Estado Nacional se tornaron imprescindibles para la continuidad del sistema ferroviario y dejaron de ser una fuente de ingresos minoritaria para pasar a ser su principal vía de sostenimiento (Instituto de Transporte Ferroviario, 2013; Melamud, 2014).

10:: En los años 90 en Argentina el conjunto de las líneas de pasajeros de la región metropolitana fue otorgado en concesión a empresas privadas (Trenes de Buenos Aires, Metropolitano, Metrovías y Ferrovías). El Estado mantuvo la titularidad de la infraestructura ferroviaria, relativa potestad sobre la toma de decisiones y pasó a asumir con mayor intensidad funciones de control de la actividad empresarial. Con este último propósito fue creada la CNRT (Decreto № 660/1996). 
Las dimensiones controvertidas en la arena pública por los actores intervinientes del debate, como la Justicia, el Gobierno Nacional, referentes de partidos opositores, sindicatos y algunos periodistas especializados en la temática, como Diego Cabot, Omar Lavieri, Diego Rojas (Hernandez y Saferstein, 2018) apuntaron a la participación de funcionarios del Gobierno, sindicalistas y empresarios en este entramado, caracterizado públicamente como "trilogía siniestra”. Dicha expresión fue resultante del desarrollo de la causa judicial que avanzó sobre las condiciones estructurales que posibilitaron que el asesinato se produjese (Centro de Estudios Legales y Sociales, 2012).

Más allá de los distintos movimientos argumentativos que los actores involucrados en la disputa pública tuvieron acerca del hecho y sus implicancias, encontraron un punto de convergencia para caracterizar al problema ferroviario. La corrupción comenzó a emerger como un elemento aglutinante de las distintas visiones que circulaban en torno a la cuestión y como elemento explicativo de su agudo deterioro. Muestra de ello es el siguiente fragmento, citado a modo de ejemplo:

Toda muerte joven es una tragedia, todo asesinato lo es por partida doble. Ninguno cobra sentido merced a acciones ulteriores, porque cada persona es única y cada vida irreparable. Pero conmociones atroces suscitan, en sociedades complejas y vivaces como la Argentina, cambios postergados [...]. El homicidio del joven Ferreyra debe, necesariamente, reavivar el debate sobre el sistema sindical argentino, la dudosa legitimidad de algunos de sus emergentes, la imperiosidad de reconocer nuevas formas de representación o agremiación, centrales alternativas. Y también desmadejar la perversa trama de la actividad del transporte de pasajeros, en la que gremialistas, funcionarios y empresarios suelen usar los tres sombreros, demasiado entreverados, demasiado cercanos (Wainfeld, 21 de octubre de 2010, Página /12).
En este camino, la reiteración de diversos accidentes asociados a la responsabilidad empresarial, debido a las implicancias que el desgaste del material ferroviario tuvo en su producción, generaron un nuevo giro en el eje de la controversia. La denominada "Tragedia de Once" llevó a la configuración del movimiento de familiares de las víctimas que tomaron protagonismo en el desenvolvimiento de los hechos. La repercusión pública del accidente y la movilización de los familiares puso nuevamente en el centro de la escena a la calidad del servicio metropolitano de trenes, introduciendo un sesgo hacia la seguridad brindada en su prestación.

En este esquema, los familiares tuvieron un posicionamiento estratégico en la jerarquía de credibilidad que afecta a los actores participantes en los debates (Langbehn, 2015; Schillagi, 2011; Loseke, 2007). Estos gozaron de una alta legitimidad social fundada en la figura de la víctima que, al estar vinculada en la historia local al terrorismo de Estado de la última dictadura cívico militar, actúa como una categoría legitimante de la acción pública (Pereyra, 2005; Pita y Pereyra, 2020). También el apoyo social ganado por el movimiento de familiares puede observarse en datos provenientes de un relevamiento propio realizado en el acto conmemorativo a los dos años del acontecimiento en Plaza de Mayo. En su mayoría, los presentes encuestados no eran usuarios frecuentes del servicio y afirmaban no tener ningún vínculo personal con los familiares y las víctimas. El principal motivo de su participación era la demanda por justicia (FVOIIGG, 2014).

También el apoyo públicamente manifiesto por parte de distintos referentes de la cultura y de la vida política dio cuenta de la relevancia social que ganó el movimiento. Pueden mencionarse al Papa Francisco y aquellos sobresalientes por su actividad en la defensa de los derechos humanos, como Adolfo Pérez Esquivel (Premio Nobel de la Paz) y Nora Cortiñas (presidenta de la Asociación Madres de Plaza de Mayo Línea Fundadora). Trabajadores pertenecientes al sindicalismo combativo, organizaciones de usuarios y otras organizaciones de familiares como las de Cromañón, Madres del Dolor y 
Agrupación por el Esclarecimiento de la Masacre Impune de la AMIA (A.P.E.M.I.A) también acercaron su solidaridad al movimiento (Hernandez, 2020). Este reconocimiento social colaboró en que instalaran su visión respecto del sistema ferroviario, encastrando las piezas del problema y configurándolo en base a la corrupción, como a modo ilustrativo se muestra en el fragmento siguiente:

La corrupción que unió a empresarios, sindicalistas y exfuncionarios del Gobierno Nacional fue la base donde se generó la tragedia previsible de Once [...] es inexplicable la inacción durante los ocho años anteriores de esta administración que acentuó y llevó al límite a una estructura ferroviaria que arrastraba años de desidia. Sobró tiempo para modificar lo que llamaban herencia recibida. Nunca quisieron hacerlo [...] ignoraron las denuncias, los avisos, cajonearon las multas, y en ese autismo político cimentaron el desastre (Menghini - Referente movimiento de familiares de Once-, 17 de febrero de 2013, La Nación).

Llegada esta etapa, los elementos emergentes con anterioridad terminaron por cohesionarse. Funcionarios del Gobierno Nacional (quienes recibieron una fuerte carga de atribución de su responsabilidad por su función), empresarios, funcionarios de la CNRT y sindicalistas fueron señalados como responsables de montar un entramado de espurio en la forma de producir el servicio que terminó por favorecer el interés privado a expensas del padecimiento de sus pasajeros. "La corrupción mata" fue la categoría que se instaló públicamente para aludir al carácter del problema, devenida en parte de lo que el caso Ferreyra sedimentara y terminando por estabilizar el proceso de construcción del problema ferroviario bajo el mencionado axioma.

"A un año de la tragedia de Once queda claro e instalado en la sociedad que la corrupción mata. La corrupción no tiene como consecuencia principal el enriquecimiento de un funcionario. Es eso, pero también mucho más" (Secchi, 24 de febrero de 2013, La Nación). En los 32 actos protagonizados por los familiares de las víctimas entre los años
2012-2015, el eje de sus documentos fueron las atribuciones a la corrupción empresaria-estatal (ACC-IIGG, 2015). La resolución de la causa donde fueron condenados 21 de los 28 imputados, destacándose entre ellos empresarios ferroviarios y funcionarios de la cartera de transporte, reforzaron la imagen socialmente construida. Finalmente, la estabilización del proceso de problematización encontró también asidero en las acciones del entonces Gobierno Nacional, observables en la promoción de cambios relevantes en el servicio ${ }^{11}$ que, vía la estatización de la mayoría de las líneas de la red, desmontaron el esquema de relaciones operante entre los actores intervinientes en su prestación.

\section{Conclusiones}

Durante el período 2002-2015, los trenes urbanos fueron objeto de controversias que involucraron la movilización de un conjunto de actores participantes en la disputa por la imposición de visiones sobre la cuestión ferroviaria. La creciente homogeneización que adquirieron las narrativas emergentes lograron consolidarlo como problema de carácter público. "La corrupción mata" fue la síntesis convergente que operó como variable explicativa del problema. Las degradadas condiciones de prestación fueron atribuidas a las relaciones de connivencia entre funcionarios del entonces Gobierno Nacional, empresarios, funcionarios del ente de control y los sindicatos, quienes -a los fines de obtener beneficios particulares-tuvieron a la calidad del servicio como variable de ajuste.

Este proceso de construcción de hegemonía de sentido fue producto de las características específicas que asumió la conflictividad. La construcción de los ferrocarriles metropolitanos como problema público -lejos de responder a una movilización colectiva orientada a demandar por mejoras en las condiciones de prestación- fue fruto de la articulación de un conjunto de componentes fuertemente disruptivos.

$11::$ Para conocer en detalle las transformaciones ocurridas en los ferrocarriles metropolitanos relativas a su forma de organización social puede consultarse Pérez y Rebón (2017). 
La violencia manifiesta en distintas expresiones como los ataques, el asesinato o la muerte masiva operaron en la capilaridad sensitiva de las distintas arenas de discusión, consolidando acontecimientos resonantes que permearon la esfera pública. La amplia legitimidad social ganada por los familiares de las víctimas de Once fue el corolario del proceso que zurció los distintos componentes presentes en las controversias.

$\mathrm{Al}$ profundizar en la reflexión en torno al caso -el hecho de que el sistema ferroviario se estructure con base en la corrupción- se ponen en consideración dos cuestiones centrales que marcan divergencias y convergencias en el modo en que en que el problema público ferroviario está anudado al problema público de la corrupción. En su amplia acepción esta última es definida como la apropiación privada de lo público, sea para la política o para el enriquecimiento personal, algo que -como muestran distintas investigaciones- constituye un problema público de larga data. El modo en que el sistema ferroviario fue concebido no puede subsumirse mecánicamente a esta definición. "La corrupción mata" da cuenta de la especificidad con que el problema se consolidó. La homologación de ambos términos estuvo estrechamente vinculada al tipo de acontecimientos sucedidos y a los actores que se movilizaron al respecto, elementos que pasaron a ser constitutivos de su estructuración. Establecidas las distancias, las proximidades abren otro nudo de atención. La puesta del interés privado sobre el público que supone un acto de corrupción está vinculado a una dimensión de apropiación económica. En los ferrocarriles metropolitanos estuvo asociada a la malversación de fondos por parte de funcionarios del Gobierno del Estado en cooperación con actores del sistema y a la laxitud de controles de los organismos competentes.

Estas variables centran la atención en las inclusiones, exclusiones y jerarquías que las problematizaciones sociales evidencian, condición frente a la que se abren interrogantes: ¿Puede la degradación del servicio reducirse meramente a una explicación anclada a una dimensión monetaria? ¿Fue solamente la falta de inversiones efectivamente realizadas en el mantenimiento del servicio lo que contribuyó a su agudo deterioro? ¿Pueden identificarse otros factores explicativos que involucren a la totalidad de los actores del sistema ferroviario que no necesariamente respondan a una lógica de aprovechamiento económico? Los procesos sociales que marquen el futuro derrotero de los ferrocarriles metropolitanos serán los que pongan en crisis o refuercen las convenciones vigentes.

Agradecimientos: Quiero agradecer a Agustina Súnico por su lectura atenta a las versiones preliminares de este artículo. Se la exime de cualquier responsabilidad respecto a errores $u$ omisiones.

\section{Referencias}

Alsina, R. M. (1989). La construcción de la noticia. Barcelona, España: Paidós.

Antón, G., Castro, J., Rebón, J., y Salgado, R. (2011). Una década en disputa. Apuntes sobre las luchas sociales en Argentina. En: M. Modonessi y J. Rebón, Una década en movimiento. Luchas populares en América Latina en el amanecer del siglo XXI (pp. 19-44). Buenos Aires, Argentina: Prometeo-CLACSO-UBA.

Basualdo, V., Morales, D., y Cabello, A. (2014). El caso del asesinato de Mariano Ferreyra y la problemática de la tercerización. En V. Basualdo y D. Morales (Coords.), La tercerización laboral. Orígenes, impacto y claves para su análisis en América Latina (pp. xx-xx). Buenos Aires, Argentina: Siglo XXI Editores.

Cabot, D. (05 de septiembre de 2008). El servicio que opera con contrato vencido. La Nación. Recuperado de http://www.lanacion. com.ar/1046827-el-servicio-que-opera-con-contratovencido

Centro de Estudios Legales y Sociales. (2012). Derechos Humanos en Argentina: Informe 2012. Buenos Aires, Argentina: Siglo XXI Editores.

Comisión Nacional de Regulación del Transporte. (2017). Serie Estadistica Ferroviaria. Buenos Aires, Argentina: Comisión Nacional de Regulación del Transporte.

Decreto 2075/2002. (16 de octubre de 2002). Declárase en estado de emergencia a la prestación de los servicios correspondientes al sistema público de transporte ferroviario de pasajeros de superficie y subterráneo del AMBA. Recuperado de $h t t p: / / s e r v i c i o s . i n f o l e g . g o b . a r / i n f o l e g l n t e r n e t / a n e-$ xos/75000-79999/78770/norma.htm 
Decreto N660/1996. (24 de junio de 1996). Administración Nacional. Reforma del estado. Modificación de actual estructura. Poder Ejecutivo Nacional. Argentina. Recuperado de: http://servicios.infoleg. gob.ar/infoleglnternet/verNorma.do?id=37574

Estación Horror. (23 de febrero de 2012). Página/12. Recuperado de http://www.ensamble19.com.ar/VERTAPAS/tapasARCHIVOarg2. php?dia=23\&tmes=2\&tanio=2012\&tsetear_tapas=Ver+Tapas

Etchemendy, S. y Collier, R. (2008). Golpeados pero de pie. Resurgimiento sindical y neocorporativismo segmentado. Postdata. Revista de reflexión y análisis político, 13, 145-192.

Frigerio, L. (1997). La construcción de problemas sociales: cultura, política y medios de comunicación. Comunicação e Política, 4(2), 137-149. Recuperado de http://www.alejandrofrigerio.com.ar/ publicaciones/religion/Frigerio_Construccion_Problemas_Sociales_1997.pdf

Fuerte conmoción por el asesinato de un militante (21 de octubre de 2010). Clarin. Recuperado de http://www.ensamble19.com.ar/VERTAPAS/tapasARCHIVOarg2.php?dia=21 \&tmes=10\&tanio=2010\&tsetear_tapas=Ver+Tapas

Furia y Caos por atraso de trenes (16 de mayo de 2007) Clarín. Recuperado de http://www.ensamble19.com.ar/VERTAPAS/tapasARCHIVOarg2.php?dia $=16$ \&tmes $=5$ \&tanio $=2007$ \&tsetear_tapas $=$ Ver+Tapas

Gusfield, J. R. (2014). La cultura de los problemas públicos el mito del conductor alcoholizado versus la sociedad inocente. Buenos Aires, Argentina: Siglo XXI Editores. (Trabajo publicado originalmente en 1981)

Hernandez, C. (2017). Entre conflictos y accidentes. La construcción de los ferrocarriles metropolitanos como problema público (20022015) (Tesis de maestría en Investigación en Ciencias Sociales). Facultad de Ciencias Sociales, Universidad de Buenos Aires, Buenos Aires.

Hernandez, C. (2020). Familiares en acción. La tragedia de Once y la cuestión ferroviaria metropolitana en la escena pública. En M. V. Pita y S. Pereyra (Eds.), Movilización de víctimas y demandas de justicia en la Argentina contemporánea (pp. xx-xx). Buenos Aires, Argentina: Teseo.

Hernandez, C. y Saferstein, E. (2018). El espacio editorial argentino y la construcción de los ferrocarriles metropolitanos como problema público. Question, 1(57). Recuperado de: http://perio.unlp.edu.ar/ ojs/index.php/question/article/view/4330/3733
Hilgartner, S. y Bosk, C.L. (1988). Therise and fall of Social Problems: A Public Arenas Model. The American Journal of Sociology, 94(1), 53-78. Recuperado de https://www.unc.edu/ fbaum/teaching/ PLSC_SOC_497_SP_2008/Hilgartner_Bosk_AJS_198 8.pdf

Instituto de Transporte Ferroviario (2013). Una estrategia para la rehabilitación de los ferrocarriles metropolitanos de Buenos Aires. Buenos Aires: Foro de discusión sobre el FC suburbano, Universidad Nacional de San Martín. Recuperado de

Kralich, S. (2016). Urbanización y transporte. Algunos aportes conceptuales. Revista Transporte y Territorio, 15, 41-67. Recuperado de http://revistascientificas.filo.uba.ar/index.php/rtt/article/view/2848

Langbehn, L.C. (2015). Arenas de conflicto y construcción de problemas públicos ambientales. Un análisis de la productividad del caso de la Ley de Bosques y del Ordenamiento Territorial de Bosques Nativos de Salta (2004-2009) (Tesis de doctorado). Facultad de Ciencias Sociales, Universidad de Buenos Aires, Buenos Aires, Argentina.

Ley 27.132. (20 de mayo de 2015). Política de reactivación de los ferrocarriles de pasajeros y de cargas, renovación y mejoramiento de la infraestructura ferroviaria, incorporación de tecnologías servicios. Declaración de interés público nacional. Buenos Aires, Argentina. Recuperado de http://servicios.infoleg.gob.ar/infoleglnternet/anexos/245000249999/247081/norma.htm

López, L. (diciembre 2008). Herramientas teóricas para el estudio de la emergencia de problemas públicos en barrios de clases medias en la Ciudad de Buenos Aires. Presentado en V Jornadas de Sociología de la UNLP. Departamento de Sociología, Facultad de Humanidades y Ciencias de la Educación, Universidad Nacional de La Plata, La Plata.

Lorenc Valcarce, F. (2005a). La sociología de los problemas públicos. Una perspectiva crítica para el estudio de las relaciones entre la sociedad y la política. Nómadas. Critical Journal of Social and Juridical Sciences, 12, 141 - 150. Recuperado de http://revistas. ucm.es/index.php/NOMA/article/view/NOMA0505220141A

Lorenc Valcarce, F. (2005b). El trabajo periodístico y los modos de producción de la noticia: el tratamiento de la inseguridad en la prensa argentina. Question, 1(7). Recuperado de http://perio. unlp.edu.ar/ojs/index.php/question/article/view/108

Loseke, D. R. (2007). Thinking about Social Problems: An Introduction to Constructionist Perspectives. New York, NY: Aldine de Gruyter

Marín, J. C. (2007). El ocaso de una ilusión: Chile 1967/1973. Buenos Aires, Argentina: Ediciones PICASO. 
Melamud, A. (2014). Subsidios y compensaciones al transporte. Asociación Argentina de Presupuesto y Administración Financiera Pública.

Menghini, P. (17 de febrero de 2013). La corrupción, base de una tragedia previsible. La Nación. Recuperado de http://www.lanacion. com.ar/1555497-la-corrupcion-base-de-unatragedia-previsible

Pereyra, S. (2013). Política y transparencia. La corrupción como problema público. Buenos Aires, Argentina: Siglo XXI Editores.

Pereyra, S. (2005). ¿Cuál es el legado del movimiento de derechos humanos? El problema de la impunidad y los reclamos de justicia en los noventa. En F. Schuster, F. Naishtat, G. Nardacchione, y S. Pereyra (Comps.), Tomar la palabra. Estudios sobre protesta socialy acción colectiva en la Argentina contemporánea (pp. xx-xx). Buenos Aires, Argentina: Prometeo Libros.

Pita, M. V. y Pereyra, S. (Eds.). Movilización de víctimas y demandas de justicia en la Argentina contemporánea. Buenos Aires, Argentina: Teseo.

Pérez, V. (2013). Estallidos de hostilidad en el transporte ferroviario de pasajeros del Área Metropolitana de Buenos Aires. 2002-2010 (Tesis de doctorado). Facultad de Ciencias Sociales, Universidad de Buenos Aires, Buenos Aires, Argentina.

Pérez, V. y Rebón, J. (Comps.). (2017). La perturbación como motor de la historia. Los ferrocarriles metropolitanos durante el kirchnerismo. Buenos Aires, Argentina: Biblos-CLACSO.

Rebón, J. y Hernandez, C. (2016). Las formas de la acción colectiva en el sistema ferroviario de pasajeros de la Región Metropolitana de Buenos Aires. Revista Transporte y Territorio, 16, 201-219. Facultad de Filosofía y Letras. Universidad de Buenos Aires.

Programa de Investigación sobre el Movimiento de la Sociedad Argentina. (s. f.). Cronologías del conflicto 1997-2016. Recuperado de http://www.pimsa.secyt.gov.ar/Crono.html

Río Ruiz, M. (2008). Usos y abusos de la prensa como fuente de datos sobre acciones colectivas. Empiria: revista de metodología de ciencias sociales, 16 .

Schillagi, C. (2011). Problemas públicos, casos resonantes y escándalos. Polis Revista Latinoamericana, 30. Recuperado de http://polis. revues.org/2277

Schuster, F., Pérez, G., Pereyra, S., Armesto, M., Armelino, M. García, A., Natalucci, A.... Zipcioglu, P. (2006). Transformaciones de la protesta social en Argentina 1989-2003. Documento de trabajo № 48. Grupo de Estudios sobre Protesta y Acción Colectiva. Recuperado de http:// gepsaciigg.sociales.uba.ar/publicaciones/documentos-de-trabajo
Secchi, P. (24 de febrero de 2013). Cuando la corrupción también mata. La Nación. Recuperado de http://www.lanacion.com. ar/1557520-cuando-la-corrupcion-tambien-mata.

Silver, B. (2005). Fuerzas del trabajo. Los movimientos obreros y la globalización desde 1880. Madrid, España: Akal.

Snyder, D. y Kelly, W. R. (1977). Conflict Intensity, Media Sensitivity and the Validity of Newspaper Data. American Sociological Review, 42(1), 105-123.

Stiberman, L. (2017). Conflictividad laboral en la Argentina: una aproximación a las metodologías del Ministerio de Trabajo de la Nación y la Central de Trabajadores Argentinos. Argumentos. Revista de crítica social, (19), 238-273.

Tragedia anunciada (23 de febrero de 2012). Clarín. Recuperado de http://ensamble19.com.ar/VERTAPAS/tapasARCHIVOarg2.php?dia $=23$ ttmes $=2$ Ctanio $=2012$ \&tsetear_tapas=Ver+Tapas

Tilly, C. (2008). Contentious performances. New York, NY: Cambridge University Press.

Tilly, C. (2000). Acción colectiva. Apuntes de Investigación del CECyP, 6, 9-32.

Tuchman, G. (1973). Making news by doing work: routinizing the unexpected. American Journal of Sociology, 79(1), 110-131.

Un tren explosivo (05 de septiembre de 2008). Página/12. Recuperado de https://www.pagina12.com.ar/diario/principal/diario/index-2008-09-05.html

Vales, L. (21 de octubre de 2010). A sangre fría. Página/12. Recuperado de https://www.pagina12.com.ar/diario/principal/index-2010-10-21. html

Viotti, N. (2015). El affaire Ravi Shankar. Neo-hinduismo y medios de comunicación en Argentina. Sociedad y Religión, 25(43), 13-46.

Vommaro, G. y Baldoni, M. (2012). Bernardo y Mariano: Las transformaciones del periodismo político en Argentina, de los años ochenta a los años noventa. Mediálogos, 2,59-81.

Wainfeld, M. (21 de octubre de 2010). La carga de la prueba. Página/12. Recuperado de http://www.pagina12.com.ar/diario/elpais/subnotas/155429-49885-2010-10-21.html.

Contribución autoral: a) Concepción y diseño del trabajo; b) Adquisición de datos; c) Análisis e interpretación de datos; d) Redacción del manuscrito; e) revisión crítica del manuscrito.

C. H. ha contribuido en $a, b, c, d$, e. 